\title{
La responsabilidad y la gestión medioambiental de la industria maquiladora Un estudio de caso
}

\author{
María Eugenia De la Rosa Leal*
}

\section{Resumen}

El estudio del comportamiento habitual con el medio ambiente es la línea de este trabajo que - con un estudio de caso a una planta maquiladora en Hermosillo, Sonora (México)revisa la operación cotidiana de los procesos de producción para establecer el tipo de gestión y responsabilidad medioambiental.

En el desarrollo del caso se establece un marco teórico compuesto por un modelo adaptado de las teorías ecológicas y el marco conceptual de la gestión medioambiental para revisar y analizar la visión medioambiental de la planta.

El estudio determina la dimensión de las variables de gestión y el impacto de ellas en la operación y retroalimentación de la misma, la forma de operación, la interacción de los sistemas y la forma de adopción de un enfoque ambiental. Se concluye con la existencia de una gestión pragmática y una responsabilidad medioambiental de naturaleza obligatoria, interesada en la disminución de riesgos sin crear valores de sustentabilidad en la planta, ni acciones de beneficio social.

Palabras clave: gestión medioambiental, responsabilidad medioambiental, organizaciones, plantas maquiladoras.

\section{Introducción}

T a dimensión de la responsabilidad en la protección del medio ambiente — como una labor compartida entre la sociedad, el gobierno y las empresas - es un argumento del debate entre la sustentabilidad y el beneficio

\footnotetext{
* Profesora investigadora de la Academia de Contabilidad Superior de la Universidad de Sonora. Correo electrónico: maedelarosa@hotmail.com
} 
económico, que se propone desde la revisión de políticas económicas nacionales hasta la instrumentación de beneficios financieros que ayuden y motiven a las organizaciones a enfrentar los diferentes niveles de compromiso con su entorno natural y lograr, así, un beneficio social.

En ese sentido, cada país ha asumido su papel de fomentar el respeto por el entorno utilizando dos criterios básicos: la emisión de reglamentos específicos y la apertura a certificaciones internacionales que implican la competencia ética de los negocios con un replanteamiento de conductas en pro del medio ambiente; no obstante, la realidad operativa de cada organización es diferente en razón de sus particularidades. El hecho es que el medio ambiente es afectado por las emisiones de gases y residuos que las empresas y particularmente las industrias generan con un efecto residual y acumulado que daña la calidad de vida y el yacimiento de los recursos naturales.

En este contexto, este trabajo estudia las variables que influyen en la conducta medioambiental de una planta maquiladora prototipo de Hermosillo, Sonora (México) para determinar el tipo y alcance de la gestión y responsabilidad medioambiental. El interés de este estudio fue conocer el grado de apropiación de la sustentabilidad en el funcionamiento cotidiano de la planta maquiladora caso como un punto de reflexión que permita determinar el compromiso empírico con el medio ambiente y su expresión.

El estudio de caso de la planta se aplicó de agosto 2003 a febrero 2004. Su conducción se dirigió para conocer el pensar y actuar de los ejecutivos responsables de las actividades medioambientales; reconocer la operación general; establecer el impacto medioambiental del proceso de producción; identificar las actividades de protección o cuidado medioambiental que se desarrollan, así como determinar de qué manera se manifiesta la responsabilidad medioambiental en el funcionamiento cotidiano. Los informantes del estudio fueron el gerente de producción, el supervisor de seguridad e higiene de la planta maquiladora y el contralor - cuyos nombres se omiten por solicitud expresa_-, quienes son los responsables de implantar o controlar cualquier tipo de acción medioambiental.

La planta maquiladora caso fue AMP Amermex en Hermosillo, la cual fue seleccionada como muestra de estudio para reconocer y establecer las relaciones existentes entre las actividades operativas de la industria maquiladora y las actividades de protección y cuidado del medio ambiente, para efectuar lo anterior se partió de la pregunta: ¿cómo se integra la responsabilidad medioambiental en la gestión de la planta maquiladora? 
La responsabilidad y la gestión medioambiental de la industria maquiladora

Un estudio de caso

El estudio de caso se apoyó en dos supuestos: 1) las actividades medioambientales son la evidencia de la responsabilidad medioambiental; y 2) la gestión medioambiental es un requisito teórico de la responsabilidad medioambiental. El estudio presenta la interpretación que hacia el interior de la planta maquiladora se da a la protección y cuidado del medio ambiente; mientras, el reconocimiento de la gestión medioambiental mostró una relación de variables como característica del desempeño ambiental.

\section{Antecedentes}

Las plantas maquiladoras, como expresión de la globalización, son utilizadas estratégicamente para disminuir costos y eficientar procesos en segmentos productivos distribuidos entre diversos países. Así, en México proliferan las plantas maquiladoras, como un sector económico emergente de la industria de exportación con sus implicaciones particulares en inversión y operación, con lo cual integran en el norte del país un corredor fronterizo del cual Sonora forma parte.

La conducta ambiental de las organizaciones ha sido estudiada en México por distintas vertientes; desde las ciencias administrativas entre 1998-2004 se encuentran dos estudios de la gestión medioambiental y sus costos. Montalvo (1992) y Lezama (2001), quienes reconocen la percepción social y administrativa de las empresas en la protección del medio ambiente, tienen en común el uso del método de estudio de caso y la teoría de la firma.

Carlos Montalvo (1992: 107) estudia el valor del costo ambiental del crecimiento industrial de las plantas maquiladoras en Tijuana B.C.; él concluye que estas plantas realizan descargas mínimas de contaminantes de manera constante cumpliendo con las reglamentaciones; sin embargo, la acumulación de contaminantes a través del tiempo representa un importante riesgo a la calidad de vida de la población y al medio ambiente; por ello, propone un plan administrativo que tome decisiones racionales con el medio ambiente que genere y use información cuantitativa de las emisiones y de las descargas generadas por las maquiladoras.

Por su parte, Cecilia Lezama (2001: 98) en Guadalajara, Jalisco, estudia las estrategias empresariales utilizadas en la innovación tecnológica ambiental de la industria fundidora; concluye que los cambios tecnológicos que internamente se realizan son los estrictamente necesarios para mantenerse en el mercado y evitar problemas de sanciones o clausuras reglamentarias de cualquier índole, sin que 
este cambio sea un comportamiento general o considere mejoras a favor del medio ambiente, como un pensamiento ambiental común de los industriales y administradores en dicha región. Montalvo y Lezama coinciden en la imprecisa existencia de una gestión o responsabilidad medioambiental en las empresas, en el noroeste y occidente del país.

Las conclusiones de los estudios anteriores establecieron una dependencia directa de las actividades y la responsabilidad ambiental con la reglamentación del país; además, existe un bajo compromiso de los empresarios con el medio ambiente y la sociedad. Lo anterior se ubica dentro de una lógica económica señalada por Hoteling (1931, citado por Costanza, et al., 1999: 49) del hecho contundente de que el interés de las empresas en la protección de los recursos naturales está en relación directa con la obtención de la mayor ganancia, por lo que las decisiones y conductas dependen de ello.

\section{Marco teórico y de referencia}

\subsection{Marco teórico}

Para este estudio fue importante la revisión del tratamiento de los asuntos medioambientales en las teorías organizacionales y la gestión medioambiental con el fin de conocer la visión de la administración en la interiorización del medioambiente y adoptar una posición teórica de estudio de los asuntos cotidianos del medio ambiente en la planta caso.

\subsubsection{Las teorías organizacionales}

Las teorías organizacionales se han visto implicadas con el medio ambiente en la medida que se ha reconocido e interiorizado en el funcionamiento de las organizaciones como una variable específica: la medioambiental. La evolución de las teorías ha seguido un camino histórico paralelo al pensamiento global de la sustentabilidad; insertándose en un primer momento como una actividad más, en la estructura burocrática tradicional a través de puestos específicos especializados o no en acciones medioambientales sin mayor distinción, situación que se dio durante lo años setenta y ochenta. 
La responsabilidad y la gestión medioambiental de la industria maquiladora

Un estudio de caso

En el inicio de los noventa, desde la visión institucional de rescate de valores, Eric Trist (1992: 316-327) habla de una nueva visión ecológica en las organizaciones, que señala la importancia de la variable medioambiental para ser atendida e investigada por su propio argumento y no tratada como una actividad común. Para esto propone crear nuevas unidades administrativas dentro de la estructura formal de la empresa para atender la ecología de la organización, entendida ésta como la interacción necesaria de la organización con la naturaleza. Esta nueva visión utiliza el enfoque de sistemas, el énfasis en la cultura interna y el uso de la tecnología para establecer y desarrollar una gestión medioambiental en la organización.

Hannan y Freeman (1992: 329-330) amplían la visión de interacción de la organización con la naturaleza, añadiendo al enfoque de Tris el análisis del impacto ecológico que identifica el riesgo medioambiental de los procesos y del funcionamiento general para determinar, así, las variables de riesgo y las capacidades necesarias de la organización para adaptar su estructura y ejercer control sobre todas las posibles causas de riesgo medioambiental. Sin embargo, una limitación en la adaptación es el hecho de que esta capacidad sólo se da en algunas unidades de la estructura con características particulares que le permiten ajustarse, producir arreglos internos de inversión e información y redistribuir recursos, lo cual reduce a unidades específicamente seleccionadas las posibilidades de adaptación.

Asimismo, Hannan y Freeman señalan que una estructura burocrática integrada como sistema -adicionada con nuevas unidades para ejercer la gestión medioambiental con el uso de tecnologías de protección ambiental — puede ayudar a implantar una cultura ecológica interna, donde un principio básico es el análisis del impacto ecológico para identificar la presencia de las variables de riesgo de perturbaciones y contingencias de origen medioambiental en las organizaciones. La interpretación del modelo integrado de esta adopción ecológica se presenta en la figura 1, el cual es el marco conceptual del estudio de caso. 


\section{Figura 1}

\section{Modelo teórico de adopción del enfoque ecológico en la organización}

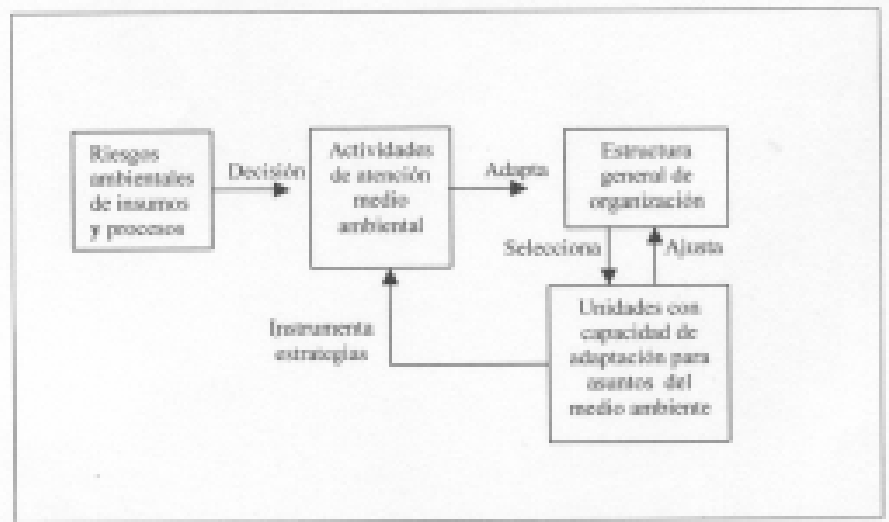

Fuente: Adaptación personal de Tris, Hannan y Freeman, 1992

El modelo de la figura 1 introduce a la empresa a entender las relaciones con sus insumos, procesos y su entorno natural a partir de sus riesgos; de forma que la interiorización de la gestión medioambiental se inicia con la determinación de actividades medioambientales para evitar o mitigar el riesgo mediante un plan de acción instrumentado por unidades administrativas flexibles con capacidad suficiente para adaptarse, ajustarse o afectarse e influir en la estructura general para estar en condiciones de atender los planes y acciones necesarias. Así, el enfoque ecológico de la organización es resultado del entendimiento de las relaciones productivas, a la vez que la gestión medioambiental presupone diferentes grados de apropiación de acuerdo con el nivel de compromiso del plan estratégico.

\subsubsection{La gestión medioambiental}

Teóricamente, la gestión medioambiental es vista como una serie de actividades y metas insertadas en los procesos de producción que implantan valores tales como el respeto y cuidado del medio ambiente al disminuir riesgos por insumos, residuos o procesos.

De esta forma, la gestión identifica los aspectos de la organización relacionados con el medio ambiente como parte de un sistema que se traduce en planes y acciones en una práctica diaria, cuyo conjunto permite distinguir el compromiso institucional con el entorno (Fernández, 2005: 156). 
La responsabilidad y la gestión medioambiental de la industria maquiladora Un estudio de caso

El sistema medioambiental opera como un plan integral de mejoras internas asistido por asesores o especialistas ambientales que evalúan continuamente los riesgos y probabilidades de impacto al medio ambiente (Thompson, 2002: 19-23). Asimismo, funciona paralelamente a otros sistemas de gestión de la organización uniendo las metas ambientales a la eficiencia de los procesos, a la vez que representa la maduración de la visión ecológica y el nivel de empate de la eficiencia económica con la eficiencia ambiental.

La gestión medioambiental es una interpretación particular de la sustentabilidad en la organización que utiliza tecnologías ambientales o adapta la tecnología estándar para la protección de los recursos y el medio ambiente; no obstante, como lo comenta Lezama (2001: 94-95), la posición sustentable es específica en cada empresa y su alcance y aplicación depende de la capacidad económica y apertura a la visión ambiental para adquirir un compromiso ecológico en el plan de negocios.

Para medir el desarrollo y dimensión de la gestión medioambiental como plan estratégico, Hunt y Auster (1990, citado por Fernández, 2005:153) establecen 5 etapas: 1) Principiante, 2) Bombero, 3) Ciudadano preocupado, 4) Pragmático y 5) Proactivo; cada una de ellas relaciona la reducción del riesgo medioambiental con el compromiso de la organización y el diseño del programa o plan medioambiental; esta correlación general de las variables de gestión medioambiental se presenta en la tabla 1.

El desarrollo de la gestión medioambiental presentada en la tabla 1 suma la conducta general de la organización en un plan teórico de trabajo como directriz de la gestión donde las variables: reducción de riesgo y compromiso de la organización están implicadas, sin que esto elimine la posibilidad de que a su vez actúen de forma emergente o en distinta dimensión.

\section{Tabla 1}

Modelo teórico de adopción del enfoque ecológico en la organización

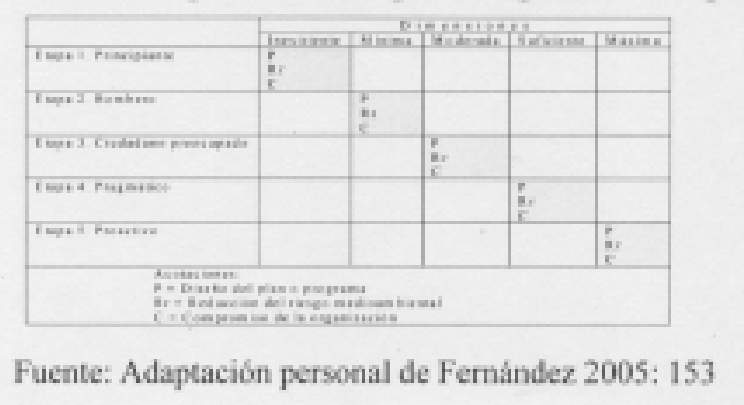


Los elementos mínimos del plan de trabajo son: objetivo, estructura de funcionamiento, informes y asesorías especializadas. El compromiso de la organización considera la complejidad de la gestión directiva, el uso de recursos financieros y el grado de involucramiento en el plan; mientras la reducción del riesgo es la disminución y control de los efectos al aire ambiente y a la salud provocados por los insumos o los procesos.

La dimensión de las variables de gestión establece la responsabilidad con el medio ambiente como una visión y posición de la organización ante el medio ambiente, resultado de conductas y programas para el cuidado de los recursos, prevención de riesgos y disminución del impacto acumulado al entorno natural.

El estudio de caso indagó las características de la gestión medioambiental en la planta caso para determinar su dimensión y con ello la responsabilidad medioambiental, calificada como inexistente, mínima, moderada, suficiente o máxima.

\subsection{Marco de referencia}

\subsubsection{Las plantas maquiladoras}

Comúnmente las plantas maquiladoras son resultado de estrategias productivas de inversión extranjera, que funcionan con un plan de trabajo y metas de eficiencia establecidas por su casa matriz, a la vez que su estructura está acoplada al modelo de organización de su grupo corporativo. Un primer acercamiento a las maquiladoras permite observar que su funcionamiento operativo y administrativo se realiza mediante una adopción obligada de rutinas de sistemas y actividades productivas, adaptadas a los requerimientos de cada línea de productos.

El por qué estudiar la industria maquiladora en Hermosillo, capital del estado de Sonora (México) obedece a la importancia de este tipo de industria en la actividad del estado porque históricamente la industria maquiladora en este lugar ha transformado la estructura económica del estado: de una economía agrícola a una economía industrial de manufactura de exportación, lo cual ha integrado a Sonora al corredor fronterizo de un norte industrializado (Contreras y Rodríguez, 2003:139); aunado a ello, está el hecho de que en el noroeste de México las plantas maquiladoras se han señalado como responsables directas de generar riesgos contra el medio ambiente y 
La responsabilidad y la gestión medioambiental de la industria maquiladora

Un estudio de caso

la salud, por el uso constante de materiales peligrosos y la generación continua de residuos tóxicos (Sánchez, citado por Montalvo, 1992). De ahí la importancia de estudiar la dimensión de la gestión medioambiental.

La industria maquiladora instalada en Hermosillo es, en su mayor parte, de manufactura de autopartes, cables para uso electrónico y componentes electrónicos, caracterizada como de segunda generación (Contreras y Rodríguez, 2003: 146) por su alto grado de especialización en los procesos de ensamble, el uso de avanzadas tecnologías, de sistemas innovadores de administración y la capacitación constante de su personal, que en su mayoría son técnicos e ingenieros altamente calificados. Cabe señalar que en su mayor parte las maquiladoras de Hermosillo son dependientes del mercado norteamericano, lo que las hace vulnerables a las crisis y contracciones de la economía norteamericana.

En materia ambiental las plantas maquiladoras son reguladas por las autoridades respectivas con actos de gobierno, reglamentos, normas ambientales y supervisiones de autoridad que controlan el uso de suelo, las emisiones a la atmósfera, las descargas a cuerpos de agua y la emisión de residuos sólidos y peligrosos (Secretaría de Medio Ambiente y Recursos Naturales, 2001), mediante la Ley General de Equilibrio Ecológico y la Protección al Medio Ambiente, las leyes complementarias: emisiones, aguas residuales y residuos, los reglamentos de las leyes complementarias; y las normas: NOM (normas oficiales mexicanas) y NMX ( normas mexicanas). El marco legal de la industria atiende dos aspectos: las emisiones ambientales y el aire ambiente laboral con acciones preventivas y correctivas; las primeras para prevenir riesgos, la contaminación y los riesgos de contingencias; las segundas para controlar, minimizar restaurar, recuperar y minimizar daños.

\section{La planta caso AMP Amermex}

El estudio de la planta AMP, Amermex en Hermosillo se realizó de agosto de 2003 a febrero de 2004 mediante entrevistas no estructuradas al contralor del parque industrial de la planta principal, al gerente de producción, al contralor de la planta y al supervisor de medio ambiente, seguridad e higiene; además, se consultaron documentos administrativos de la planta y se efectuó la observación directa del funcionamiento de dos líneas de producción. Asimismo, este estudio particular de la planta — como caso típico de las maquiladoras ubicadas en Hermosillo, Sonora- 
desarrolló un análisis a profundidad de los procesos y acciones que intervienen en el objeto de estudio: la conducta ambiental y las relaciones de gestión y responsabilidad relacionadas.

El contenido de esta sección presenta la descripción de la planta caso y su contexto como resultado de la información obtenida del análisis de documentos y las notas de campo de las entrevistas y observaciones.

\subsection{Generalidades}

AMP, Amermex, S.A. de C.V. — fundada el 15 de septiembre de 1941 en Newark, New Jersey, cuyas iniciales corresponden a Aircraft Marine Products (nombre original reducido actualmente a sus siglas) — es una planta maquiladora representativa de las industrias maquiladoras de exportación en la ciudad de Hermosillo, Sonora (México) por el número de empleos que ofrece: cerca de 3,000 en el 2005 y más de 6,000 en 1998 (fecha de mayor auge). Físicamente se ubica en dos parques industriales y ocupa el 70\% de los mismos. La planta tiene una antigüedad en la localidad de más de 17 años, tiempo poco común en el horizonte de vida de las maquiladoras. AMP, Amermex.

En 1999 AMP se integra a Tyco Internacional Ltd., División Electronics, su corporativo en Estados Unidos de Norteamérica (USA), cuya actividad general es la fabricación e instalación de sistemas de seguridad para incendios, servicios electrónicos de seguridad, material de empaque, componentes eléctricos y electrónicos.

AMP como grupo filial cuenta con 50 plantas en diferentes países alrededor del mundo que producen terminales, fibra óptica, tableros de circuitos, sensores, cables, herramientas, switches, máquinas y conectores; todo lo anterior empleado para computadoras, equipos de oficina, vehículos de transporte, instrumental médico de precisión, automóviles, aviones, equipo industrial, equipo de comunicaciones y equipo militar aéreo espacial. Los principales clientes del grupo Tyco corporativo de AMP son Intel, General Electric Corporation, Alcatel, Volkswagen, Siemens A.G., IBM Corporation, Motorola, Compaq, Iomega y Seros (AMP, 1999).

Cuando la empresa inició sus operaciones en Hermosillo dependía de un shelter (administrador de maquilas), que dejó de administrarla en 1995. La decisión de separarla del shelter fue una estrategia de su corporativo para estimular la competencia por calidad y precio entre las plantas similares AMP México, China e India con la idea de hacerlas responsables de su eficiencia y costos. 
La responsabilidad y la gestión medioambiental de la industria maquiladora

Un estudio de caso

AMP, Amermex en México — situada en el casco urbano de la ciudad de Hermosillo, la cual cuenta con tres plantas industriales con una extensión total de 680,000 pies cuadrados y en dos parques industriales, ambos al noroeste de la ciudad: uno con salida hacia el aeropuerto de Hermosillo y otro con salida hacia la frontera norteamericana- es una planta de ensamble de calidad mundial que inicia sus operaciones en diciembre de 1986 con 13 operadores en un edificio; actualmente, trabaja con 2,250 operarios en dos turnos por siete días a la semana, parando las máquinas en horario nocturno. Los turnos y el número de operarios son flexibles, pues pueden cambiar de un día para otro dependiendo de las necesidades de las líneas de producción (Valenzuela, 2003).

La industria maquiladora AMP, AMERMEX en Hermosillo se dedica al ensamble de cables electrónicos, funciona con las características propias de la industria de exportación: capital de inversión extranjero, instalada como área de ensamble en México, contrata gran cantidad de mano de obra con un alto grado de exigencia en eficiencia y tiempo en sus procesos de producción. Además, bajo un régimen fiscal de devolución temporal de insumos y mercancías al extranjero, así como jugando un importante papel en el ajuste social y económico de la región por la cantidad de empleos locales que ofrece. Cabe aclarar que la planta ubicada en México tiene a su favor los bajos costos de distribución hacia los Estados Unidos de Norteamérica, punto de entrega de sus productos, pero como desventaja los costos nacionales laborales y fiscales.

Los empleos directos de la planta son fluctuantes. En 1995 eran de 6,700 -65\% del empleo total de las maquiladoras en Hermosillo_-; en 1998 bajó a 6,000; en enero de 2004 bajaron a 4,200; al finalizar la información de este estudio febrero de 2004 bajaron a 2,250 operarios. Estas fluctuaciones de empleo son comunes por los ajustes a la demanda de producción, la eficiencia de la planta y el impacto de las recesiones económicas nacionales y norteamericanas.

AMP se ha distinguido en la localidad por su interés en los empleados y el medio ambiente por las acciones con beneficio comunitario que ha realizado, entre ellos destaca: el establecimiento y coordinación de un centro de atención infantil con distintos horarios al servicio de los trabajadores del parque industrial, la atención inmediata de los conflictos laborales, el cuidado responsable de su aire ambiente laboral y la protección de su parque industrial como parte de los comités de vigilancia y control del mismo, oponiéndose en años recientes a la instalación de tanques contaminantes expuestos al ambiente natural (Valenzuela, 2003). 


\subsection{Funcionamiento}

El funcionamiento deAMP, Amermex en Hermosillo, es el típico del régimen de maquilas: recibe materia prima importada, ensambla conforme a las indicaciones y retorna los productos y sobrantes de materia prima vía exportación al país de origen. La planta es una industria $100 \%$ de exportación, opera con estudios de transferencia y con precios de competencia con empresas similares, reporta mensualmente sus utilidades a su oficina matriz en USA y tiene como único ingreso la venta de sus productos al grupo.

AMP México orienta sus esfuerzos de eficiencia hacia el control de sus centros de costos de producción, evita la doble tributación y agiliza el exceso de requerimientos aduanales de importación y exportación para lo cual cuenta con personal altamente calificado en producción, administración y especialistas aduanales. La planta tiene la certificación ISO 9000 y el reconocimiento de Industria Limpia, otorgado por la Procuraduría Federal de Protección al Ambiente.

Su organización es por funciones, se divide en áreas integradas como sistemas; sus áreas operativas están estructuradas alrededor de las necesidades de producción. Las áreas se agrupan en gerencias funcionales: operación, administración y supervisión atendidos por el director de operaciones, contralor y gerente de planta, respectivamente. Todos los puestos están definidos por posiciones jerárquicas con su zona de poder y competencia asignada. El criterio de asignación de los puestos se basa en la subdivisión de funciones de operación, responsabilidad y control, como un modelo burocrático de delegación de funciones (Merton, s/f: 275), compuesto por varios puestos subordinados coordinados por ingenieros y personal especializado con actividades específicas relacionadas con las líneas de producción (ver organigrama general de AMP, Amermex en la figura 2).

\section{Figura 2}

\section{Organigrama general maquiladora AMP, Amermex, Hermosillo}

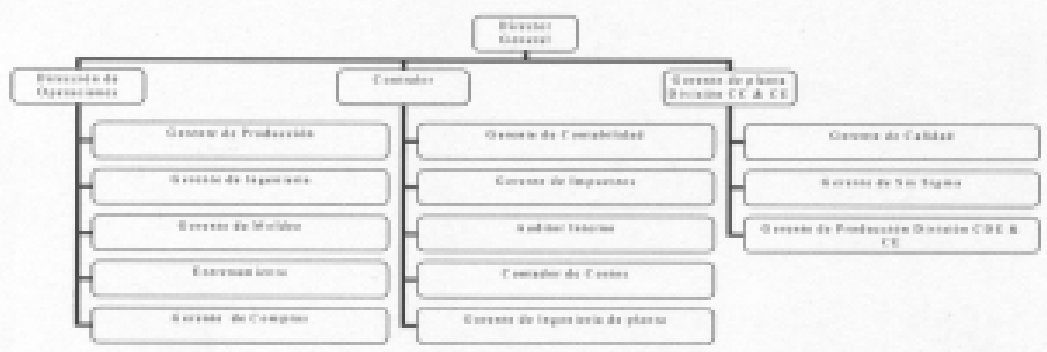

Fuente: Archivos de la industria maquiladora AMP, Amernex en Hermosillo, julio 2003 
La responsabilidad y la gestión medioambiental de la industria maquiladora Un estudio de caso

El organigrama de la figura 2 presenta tres niveles: dirección, áreas y gerencias. El organigrama total de la planta cuenta con siete niveles de autoridad, más tres niveles de empleados y operarios con una división sistemática del trabajo para lograr la eficiencia de la organización.

AMP utiliza una estructura matricial debido a que existen puestos subordinados a varios jefes (Davis y Lawrence, 1982 citado por Pariente, 2000: 134), las gerencias actúan como líderes de intervención con otros puestos. Las interacciones jerárquicas utilizan un método de supervisión y gerencia compartida para asegurar la calidad, es el caso de la gerencia de ingeniería y gerencia de calidad.

La operación de la planta es por sistemas, caracterizada por la especialización de su personal que da complejidad a su estructura (Bertalanffy, 1995: 54,163). Opera como sistema abierto por la interacción con su casa matriz con las autoridades locales y con las otras plantas de los parques industriales en que se ubica, con estas últimas bajo un plan coordinado de ordenamiento del parque industrial, que incluye acceso restringido, el manejo de residuos sólidos, depósitos de basura común, imagen pública, y vías de carga y descarga.

La relación sistémica entre las áreas se define por las conexiones (sinergias) de trabajo entre todas las áreas por el estilo de manufactura de las líneas de ensamble, situación que une el modelo mecanicista del proceso con el modelo de sistemas.

\subsection{Actuación medioambiental}

La planta está certificada como Industria Limpia, lo cual implica que cumple con todas las normas ecológicas y de seguridad e higiene establecidas por la Secretaría de Salud, Secretaría del Medio Ambiente y Recursos Naturales, Secretaría del Trabajo y Previsión Social y Secretaría de Comunicaciones y Transporte. Esta constancia de cumplimiento se otorga por la ausencia de observaciones ambientales negativas en las auditorías ambientales de ley que periódicamente se le aplican a la planta.

La gestión medioambiental de AMP está compuesta por acciones de cumplimiento de normas oficiales, que se inician en la contraloría como un plan colectivo de responsabilidades de operación y supervisión. El puesto clave del desempeño ambiental en esta planta maquiladora es el Supervisor de Medio Ambiente Seguridad e Higiene -dependiente de la Gerencia de Ingeniería de planta- con una triple función: 
atender las necesidades de seguridad laboral de la manufactura con rondas constantes supervisando la seguridad y condiciones de la planta; el seguimiento documentado de las medidas medioambientales y de seguridad tomadas y la salvaguarda de la documentación de manejo de residuos, su confinamiento, las auditorías ambientales aplicadas y las mediciones de sus emisiones a la atmósfera.

Los puestos que atienden la protección al medio ambiente son los mismos que realizan la supervisión y controlan la seguridad e higiene laboral: Medio Ambiente Seguridad e Higiene (MASH), Gerencia de Compras y sus auxiliares, Gerencia de Producción, y Gerencia de Ingeniería de planta.

Por lo que respecta al impacto ambiental de la planta, son bajas cantidades de emisiones de partículas sólidas al aire, monóxido de carbono, residuos sólidos y residuos peligrosos de aceites residuales, substancias corrosivas, inflamables, tóxicas y escorias de materia primas.

El plan de acciones de protección medioambiental y seguridad laboral considera: estaciones de trabajo con purificadores de aire, manejo integral de residuos, comunicaciones de riesgo, capacitación para el manejo de herramientas, estudios ergonómicos y comisiones de seguridad e higiene. La planta tiene además un plan de medición y estimación de emisiones, residuos y descargas de aguas residuales, que registra en bitácoras especiales, y contrata con regularidad servicios externos de auditoría ambiental y monitoreos ambientales. En relación con sus acciones de prevención, la planta ha adaptado sus equipos para controlar sus emisiones de partículas sólidas y residuos; en los procesos cuida disminuir la contaminación por emisiones y residuos; y, en algunas líneas de producción, se utilizan máscaras personales de protección visual y auditiva para gases y escorias.

Cabe señalar que existen áreas especiales en la planta para concentrar y controlar sus scraps o residuos y para almacenar los químicos que se utilizan en los procesos. El personal de scraps concentra todos los residuos sólidos, empaques, cilindros de seguridad y contenedores de residuos peligrosos de las áreas de manufactura. Esta área es parte de manufactura y está controlada por MASH, en ella el personal especializado se encarga de separar la basura general, el residuo que debe ser devuelto por exportación incluyendo envases y carretes, así como los residuos peligrosos. 
La responsabilidad y la gestión medioambiental de la industria maquiladora

Un estudio de caso

AMP tiene un plan de manejo integral de residuos: almacena temporalmente los residuos, los cataloga, empaca y realiza un diagnóstico CRETIB (corrosivo, reactivo, explosivo, tóxico, inflamable y biológico infeccioso). La mayor parte de sus residuos son basura común; recicla el cartón y la madera de los empaques y asigna valor a las escorias y envases que se devuelven a su país de origen. Los residuos peligrosos que desecha son transportados y confinados en sitios especiales dentro y fuera del país, conservando la responsabilidad de ellos por posible impacto al ambiente durante diez años.

Así, la gestión medioambiental opera virtualmente en la estructura organizacional de AMP como un mecanismo de seguridad interna del ambiente laboral y una serie de procedimientos legales y de calidad. Ligando las interacciones de la Dirección de Operaciones con la Gerencia de Producción y la Gerencia de Calidad.

Periódicamente MASH informa sobre el desempeño ambiental de la planta a las áreas de producción y a dependencias oficiales con los datos que captura y controla sobre sus emisiones, descargas, residuos, ruido y aire ambiente laboral. Comúnmente estos datos son de uso interno, no es información conocida por el corporativo, es de uso confidencial y sólo es utilizada por los puestos que deciden o intervienen en el desempeño ambiental; esta situación concentra el posible sistema ambiental en tres posiciones jerárquicas: Gerencia de Producción, Gerencia de Ingeniería de Planta y Supervisor de Medio Ambiente Seguridad e Higiene.

AMP, Amermex cuenta con la certificación ISO 9000, sin embargo, la certificación de la norma ISO 14000 medioambiental como paso lógico de las normas ISO no le interesa y argumenta que esta norma no le otorga un beneficio real por ser una norma de imagen de venta, además su adopción y mantenimiento es costoso; le es, por tanto, innecesaria porque la planta sólo requiere asegurar la calidad y disminuir sus costos como imagen de competencia intergrupo.

\section{Análisis del estudio}

A partir de los elementos de un sistema medioambiental como propuesta de análisis del caso, se parte de una concepción inicial del compromiso de la planta mediante un análisis o evaluación del riesgo ambiental, establecimiento de políticas y planes ambientales, implantación de acciones, supervisión del riesgo, corrección y retroalimentación del compromiso y del plan, con dos alternativas de implantación del sistema: por reconocimiento interno o por el interés de reconocimiento de una entidad externa dependiente de una norma emitida (Masoliver, 2000: 15-29). 
Con lo anterior, la integración del sistema de gestión en la estructura se visualiza conforme al marco teórico como un plan integral de mejoras que evalúa continuamente los riesgos ambientales (Thompson, 2002) y se integra al sistema general de organización para llevar a cabo la política medioambiental (Masoliver, 2000: 15).

\subsection{Compromiso de la planta}

Como anteriormente se señaló, AMP, Amermex es una planta maquiladora de segunda generación, caracterizada por personal calificado - directivos, ejecutivos, supervisores, operarios y técnicos - ; alto grado de especialización tecnológica; empleo de equipos y procedimientos de punta en sus procesos; y la manufactura de ensamble de cables de alta precisión. El grado de ajuste de la planta para atender sus responsabilidades ambientales se resumen en tres puntos: 1. La estructura de AMP, Amermex opera con un modelo burocrático y una estructura matricial con un puesto de supervisión (MASH) de las medidas y acciones medioambientales; 2. La planta puede tomar decisiones relacionadas con su administración interna y con la disminución de sus costos sin afectar la calidad de sus productos y las instrucciones de producción recibidas, atendiendo preferentemente la seguridad laboral; y 3. La posibilidad de invertir en maquinaria o equipos con tecnología de protección medioambiental es limitada porque la nave industrial es adaptada por el arrendador de la misma y los equipos son recibidos ex profeso con el diseño completo de cada línea de producción y devueltos al corporativo cuando la misma se concluye.

Las actividades medioambientales de AMP, Amermex son atendidas de forma compartida por la Gerencia de Producción, el supervisor de Medio Ambiente Seguridad e Higiene y el gerente de Ingeniería de planta, puestos que deciden y establecen las acciones medioambientales estrictamente necesarias en el proceso para prevención de riesgos y por las normas oficiales ambientales y las normas ISO en un enfoque de responsabilidad social como obligación legal (Chiavenato, 2006: 529).

Con las anteriores consideraciones el tipo de compromiso encontrado en la planta conforme la tabla 1, se encuentra en ciudadano preocupado porque su motivación para realizar acciones medioambientales está dirigida moderadamente por la seguridad de sus empleados, la calidad de sus productos, el interés de no incrementar sus costos y no afectar su imagen de "hacer lo correcto" como imagen social de responsabilidad y competitividad. 
La responsabilidad y la gestión medioambiental de la industria maquiladora

Un estudio de caso

\subsection{Análisis de riesgo ambiental de la planta.}

El producto principal de ensamble de la planta son los cables de uso electrónico y eléctrico para aparatos de precisión y calibrajes de alta tecnología, trabajando con tres líneas de ensamble, machine shop y moldeo, produciéndose al mismo tiempo entre 4 o 6 tipos de cables; la planta, además de los cables, produce conexiones y terminales; cada línea de cables tiene sus características particulares con pequeñas diferencias en razón de las especificaciones del cable con un proceso general que se rescata en la figura 3.

Figura 3

Análisis de impacto ambiental del proceso general de producción de AMP, Amermex

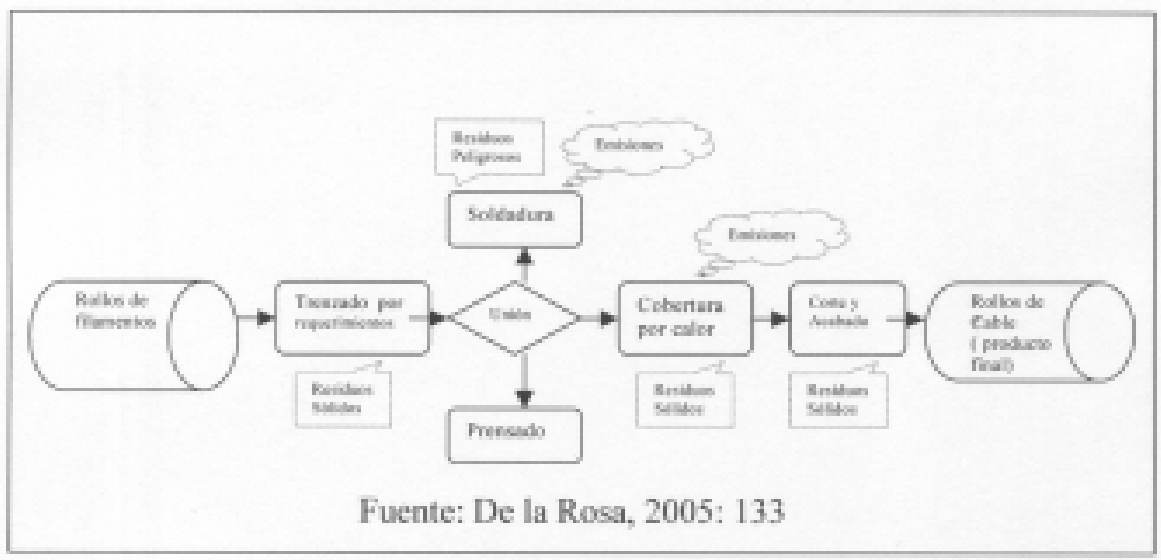

La producción de la maquiladora AMP, Amermex tiene tres procesos básicos: el trenzado, la unión y el recubrimiento; algunos cables son prensados y otros soldados, los de mayor impacto al ambiente son los de soldadura de estaño que generan emisiones de monóxido de carbono, aceites residuales y partículas sólidas de estaño; la cobertura por calor produce también monóxido de carbono al aire ambiente laboral y el riesgo por uso de químicos inflamables y explosivos. Las medidas de protección laboral adoptadas en la línea son principalmente guantes y mascarillas.

La línea de proceso de la planta es flexible por su rápida conversión con cambio de materiales, equipos, tiempo de proceso y terminados para fabricar diferentes tipos de cables con impacto diferente, que en términos generales está calificado como de bajas emisiones y generación mínima de residuos sólidos y peligrosos sin rebasar los máximos legales. 


\subsection{Políticas y planes ambientales}

El plan de operación es decidido por los recorridos a la planta y las observaciones de la gerencia de producción para evitar riesgos, contingencias ambientales y riesgos industriales; además, funciona paralela a la gestión administrativa y de producción.

El puesto clave de decisión de las acciones medioambientales es la Gerencia de Producción; éstas son actividades complementarias de protección y corrección medioambiental y se definen en razón de la disminución del riesgo laboral.

Las relaciones medioambientales encontradas en la maquiladora AMP, Amermex indican que la gestión se asume incluida en la estructura formal de la planta supervisada por Medio Ambiente Seguridad e Higiene e incluye la seguridad laboral de la planta, la atención de las observaciones de producción relacionadas con sus emisiones y residuos y el cumplimiento de las reglamentaciones ambientales; estas relaciones se representan en la figura 4.

Figura 4

Relaciones medioambientales encontradas en la planta maquiladora AMP, Amermex en Hermosillo

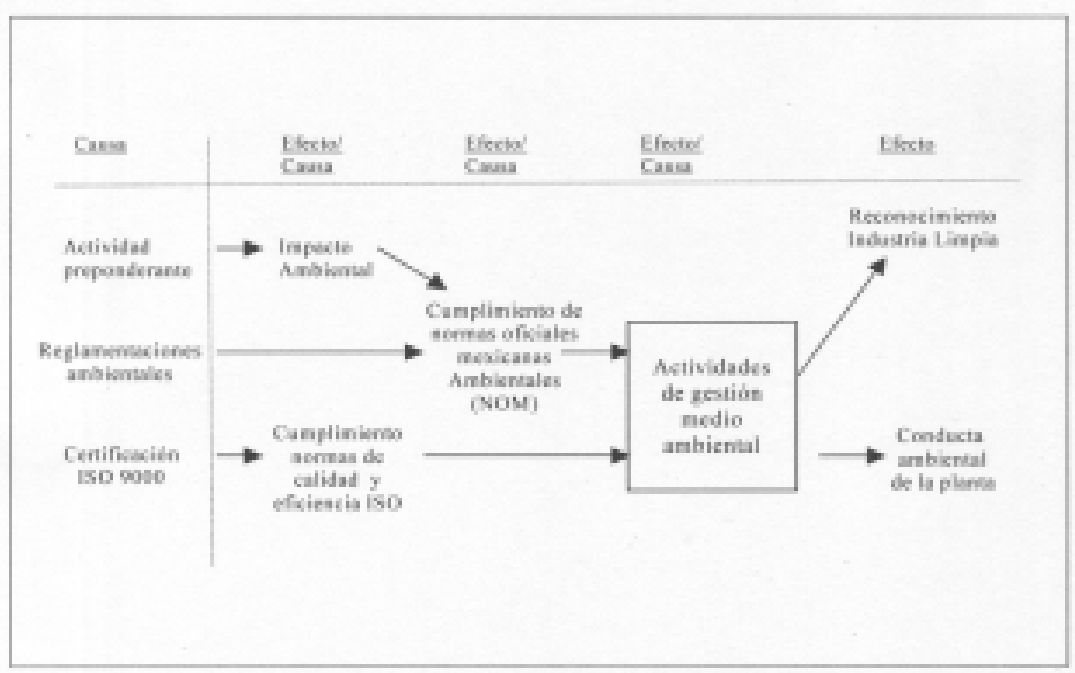

Fuente: Elaboración propia, análisis de resultados estudio de caso 
La responsabilidad y la gestión medioambiental de la industria maquiladora

Un estudio de caso

En la figura 4 se observa que las relaciones medioambientales en la planta surgen de los riesgos del proceso de la actividad preponderante, las reglamentaciones ambientales y el mantenimiento de la certificación ISO 9000. A partir de lo cual se ajustan o establecen actividades para atender el impacto ambiental y cumplir con los lineamientos de calidad y no rebasar los límites indicados en las normas NOM para conservar el reconocimiento de Industria Limpia y la certificación ISO 9000. La suma de acciones integran el plan de gestión medioambiental con la política de reducción de riesgos.

Las relaciones encontradas producen dos efectos conductuales en la planta: 1) la observancia de las disposiciones reglamentarias ambientales con uso de equipos de seguridad, controles y supervisiones rutinarias; y 2) la disminución de desperdicios.

Confrontando la actuación de la planta AMP, Amermex con el modelo teórico del enfoque ecológico (figura 1) se observa que la planta habilita el puesto del Supervisor de MASH para la gestión medioambiental, quien instrumenta las acciones medioambientales necesarias y tiene como política de actuación: la prevención y atención de riesgos al personal, el control de contingencias internas como incendios o explosiones y el riesgo en el manejo de residuos.

MASH se inserta en la estructura, dependiendo de la gerencia de producción y la gerencia de planta sin que su actuación influya en arreglos internos de inversión y redistribución de recursos para la protección del medioambiente, actuando como ejecutor de acciones específicas. Los cambios en la estructura no son comunes y son sólo aquellos que benefician la eficiencia de la producción o disminuyen riesgos por insumos peligrosos, por lo regular son procedimientos que no afectan la estructura.

\subsection{Implantación y supervisión}

La instrumentación de todas las actividades, incluyendo las medioambientales, es capacitada y realizada como rutina por el personal de la planta; de la misma forma, las posibilidades de riesgo son capacitadas y ensayadas como simulacros por las comisiones de seguridad de supervisores y operarios.

La eficiencia es una variable muy importante en la planta, ésta se mide por la relación: tiempo de entrega, calidad y costos de productos, trilogía que es ajustada por el ahorro de cualquier tipo de acción innecesaria en los procesos de producción conforme las instrucciones de los clientes. Así, las adecuaciones se deciden y establecen por la observación constante de emisiones y residuos en la línea de 
producción. Las acciones más comunes son los controles, remediaciones y tratamientos medioambientales para evitar rebasar los límites ambientales autorizados y mantener el reconocimiento de Industria Limpia.

Por lo que respecta a la implantación de las rutinas medioambientales, es importante señalar que no se detecta el fomento de valores medioambientales, la cultura presente es de prevención de riesgos laborales.

La atención general de la planta es supeditada a los requerimientos de producción y a las obligaciones legales, interpretando su filosofía de "hacer lo correcto" como una política general de actuación que se refuerza constantemente entre el personal de todos los niveles.

\section{Resultados}

\subsection{Gestión medioambiental}

Con el manejo empírico de la planta de un compromiso, análisis de riesgo, políticas y planes e implantación de medidas medioambientales, se establece la existencia de una gestión medioambiental, cuyo desarrollo y dimensión se encuentra entre las etapas: 3 moderado y 4 suficiente (tabla 1 ).

Por su parte, la gestión medioambiental se ejerce como una serie de rutinas incluidas en otras rutinas del proceso de producción de una organización burocrática, con una serie de actividades que se resumen en la figura 5 . 
La responsabilidad y la gestión medioambiental de la industria maquiladora

\section{Figura 5}

\section{Gestión medioambiental de la planta maquiladora AMP,Amermex}

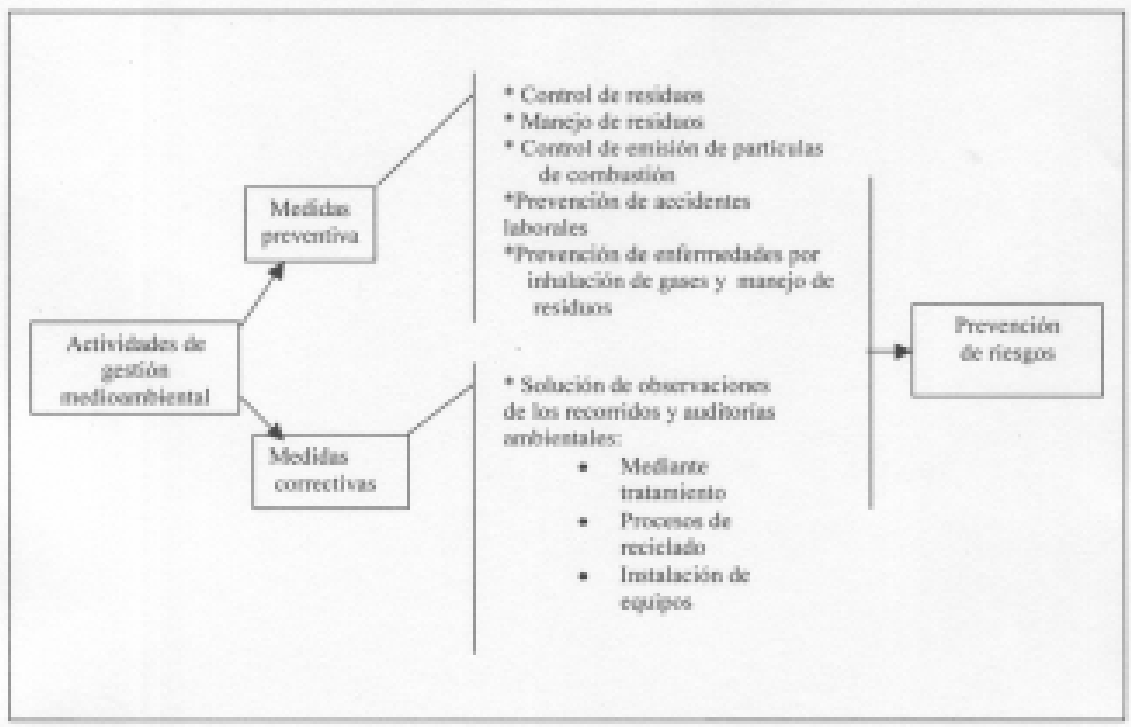

Fuente: Adaptación de De la Rosa, 2005: 137

Las actividades medioambientales de la figura 5 son de dos tipos conforme las normas NOM: medidas preventivas y correctivas. Como preventivas están el control de residuos sólidos; residuos peligrosos; emisiones de partículas de combustión; almacenaje, transporte y manejo de residuos sólidos y peligrosos; prevención de accidentes laborales; y prevención de enfermedades en personal por gases y residuos. En cuanto a las correctivas como inversión en reingeniería de procesos, reciclaje y equipos ambientales sólo se realizan si son estrictamente necesarias y no afectan los costos. Las responsabilidades contingentes por confinamiento de residuos peligrosos se establece en los documentos de depósito que establecen el compromiso legal de reparación de daño ambiental en caso de darse.

La gestión medioambiental está ubicada en las rutinas de producción de forma emergente; sin embargo, no existe hacia el interior de la planta una concepción precisa de la misma que se considera símil a la seguridad laboral. Esta significación interna es una importante barrera de la comprensión social del desarrollo sustentable y su responsabilidad medioambiental. 
Los informes medioambientales que se realizan son rutinas de control de conceptos medioambientales, establecidas por dos niveles jerárquicos - Gerencia de Producción y Gerencia de Ingeniería de planta - en un modelo burocrático de división del trabajo, apoyado por comités de calidad que cuidan la eficiencia en costos. Los datos ambientales son controlados en unidades físicas tales como partículas por millón, toneladas, mg, etc. relacionados en los informes de desempeño ambiental presentados requeridos por diversas autoridades, entre ellas se encuentra la Procuraduría Federal de Protección al Ambiente.

\subsection{Responsabilidad medioambiental}

AMP se percibe a sí misma como respetuosa de "todas" sus obligaciones y responsabilidades legales, integrando su interpretación particular de responsabilidad con el medio ambiente con una interacción como se presenta en la figura 6.

\section{Figura 6}

Comportamiento medioambiental de la planta maquiladora AMP Amermex, Hermosillo

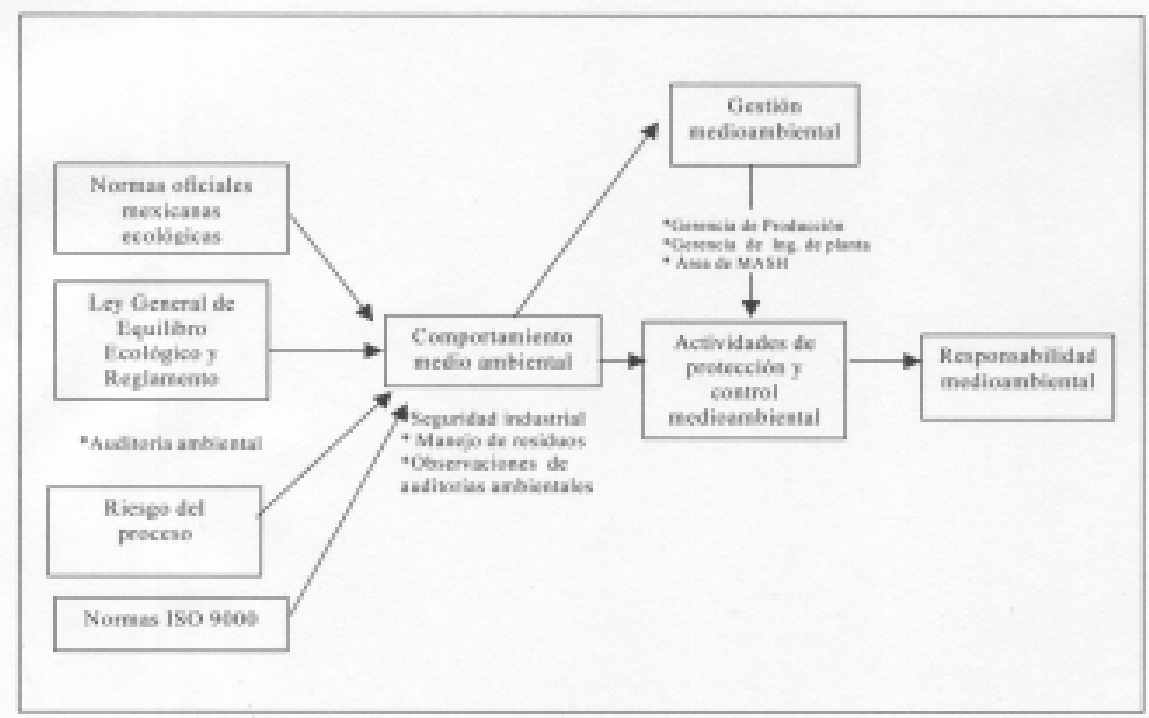

Fuente: Adaptación de De la Rosa, 2005: 136 
La responsabilidad y la gestión medioambiental de la industria maquiladora

Un estudio de caso

La conducta medioambiental como se observa en la figura 6 tiene como dirección los reglamentos, normas y riesgos del proceso; estos últimos atendidos dentro de los parámetros legales y de calidad, coordinados por la estructura de decisión de las Gerencias de Producción, de Ingeniería de planta y MASH para dar como resultado su interpretación de responsabilidad medioambiental obligatoria.

Bajo el precepto de "hacer lo correcto", la planta considera que su desempeño ambiental es adecuado, lo cual se confirma con el reconocimiento que posee como Industria Limpia, sujetándose a no rebasar los límites de polución autorizados por ley; sin embargo, la asunción de actuar bien no contribuye a una propuesta de transición de la planta hacia la sustentabilidad.

En este modelo de responsabilidad, la imagen pública de la planta no influye, tampoco se introducen valores de protección del medio ambiente; el énfasis se da en la calidad y la seguridad en los procesos. El compromiso medioambiental de la planta no considera una apropiación de una cultura medio ambiental, ni un rescate de valores ecológicos, sino atiende los aspectos obligatorios mínimos y necesarios

A pesar de que no existe una cultura de protección medioambiental en AMP, muchos de los conceptos medioambientales son familiares para los gerentes y responsables de control de diferentes niveles de la planta, pero sólo se reconoce y distingue la visión medioambiental de las reglamentaciones y los riesgos del proceso sin aportaciones adicionales al plan de gestión por parte de la planta.

\section{Conclusiones}

Es importante aclarar que este estudio de caso no tuvo como objeto evaluar ni establecer el grado de contaminación de la industria maquiladora, sino las conclusiones establecen el modo de gestión y responsabilidad medioambiental de la planta caso:

1. El análisis de riesgo ambiental de los procesos que desarrolla son calificados como de bajo riesgo conforme las disposiciones ambientales nacionales, no mediando el compromiso social para la evaluación del riesgo por el impacto acumulado en la comunidad. 
2. El compromiso ambiental de la planta es de naturaleza legal, al cuidarse como puntos focales no rebasar los límites permitidos, controlando esto con las auditorías de ley, el monitoreo de emisiones y residuos y la bitácora de control. Este compromiso no presenta propuestas adicionales de compromiso social o con el medio ambiente, se ajusta su ética de hacer lo correcto.

3. La política medioambiental se involucra dentro de la prevención de riesgos con un apego a la seguridad laboral y el cuidado del aire ambiente laboral. Ninguna de las acciones adicionales de riesgos del proceso están fuera de este marco de trabajo.

4. La gestión medioambiental se encuentra como actividad pragmática que se inserta entre el sistema de calidad y el sistema de producción sin formar un sistema medioambiental. La atención del medio ambiente tiene metas de seguridad, lo cual permite concluir que el medio ambiente no existe como variable influyente en el compromiso social de la planta.

5. El énfasis de control es sobre las emisiones y los residuos a través de la implantación de rutinas sin que otros conceptos ambientales como aguas residuales y ruido perimetral o interno tengan atención especial.

6. La cultura interna de operación no contiene valores ambientales, para ello se interpretan las conexiones operativas con las emisiones o residuos como actividades rutinarias de seguridad. La instrumentación de valores es hacia la honestidad, la calidad y los bajos costos como elementos importantes en la competitividad de la planta.

7. Por último, la responsabilidad medioambiental de la planta, como una responsabilidad social, no existe. La interpretación encontrada se remite a un compromiso de higiene y seguridad sujeto a disposiciones legales e instrucciones de su corporativo; por lo tanto, es una responsabilidad obligatoria con metas económicas sin esfuerzo adicional para conseguir una imagen social comunitaria.

La conclusión general es el hecho de que la responsabilidad y la gestión medioambiental de la planta estudiada se encuentran en una etapa y dimensión de nivel moderado y suficiente con una visión pragmática de mínimos por cumplir, 
La responsabilidad y la gestión medioambiental de la industria maquiladora

Un estudio de caso

que no promueve la apropiación del concepto social de la sustentabilidad, situación que se presenta en los estudios de Baja California (Montalvo, 1992) y Jalisco (Lezama, 2001). Dicho concepto social, puede ser ampliado con sistemas administrativos que demuestren el beneficio comunitario y económico para la organización.

\section{Fuentes de consulta}

AMP Amermex (1999), AMP Amermex folleto de inducción, Departamento de entrenamiento, Hermosillo, México.

BERTALANFFY, L. V. (1995), Teoría general de los sistemas, Fondo de Cultura Económica, México.

CHIAVENATO, I. (2006), Introducción a la teoría general de la administración, $7^{\text {a }}$ edición, Mc Graw Hill, México.

CONTRERAS, Ó. F. y J. Rodríguez Gutiérrez (2003), "La conexión del desierto: industria electrónica y proveedores globales en Sonora", en Hecho en Norteamérica, coordinado por Óscar F. Contreras y Jorgem Carrillo, Ediciones Cal y Arena, El Colegio de Sonora, México, pp. 139-163.

COSTANZA, R., et al. (1999), Una introducción a la economía ecológica, Compañía Editorial Continental, México.

DE LA ROSA LEAL, M. E. (2005), Determinación de un modelo de costos medioambientales contables para la industria maquiladora de Hermosillo, Sonora, Tesis de doctorado inédita, Universidad Autónoma de San Luis Potosí, México.

FERNÁNDEZ GAGO, R. (2005), Administración de la responsabilidad social corporativa, International Thomson Editores, Madrid.

HANNAN, M. T., y J. Freeman (1992), "The population ecology of Organizations", en Classics of Organization Theory, compilado por Jay M. Shafritz y Ott J. Steven, 3a. ed., Brooks Cole Publishing Company, Beltmont, California, pp. 329-341. 
LEZAMA, C. (2001), "Estrategias empresariales para la innovación tecnológica y la protección ambiental: el caso de una empresa fundidora",en Innovación tecnológica y medio ambiente, compilado por Leonel Corona y Ricardo Hernández, Fundación Friedrich Ebert Stiftung, pp. 79-98.

MASOLIVER JORDANA, D. (2000), Guía práctica para la implantación de un sistema de gestión ambiental. Manuales de ecogestión, Generalitat de Catalunya, España.

MERTON, R. K. (s/f), "Estructura burocrática y personalidad", en Teoría y estructura sociales, Fondo de Cultura Económica, México, pp. 275-286.

MONTALVO CORRAL, C. (1992), Costo ambiental del crecimiento industrial: el caso de la maquiladora eléctrica en Tijuana, B.C., Fundación Friedrich Ebert Stiftung, México.

PARIENTE FRAGOSO, J. L. (2000), Teoría de las organizaciones. Un enfoque de metáforas, Universidad Autónoma de Tamaulipas, México.

SECRETARÍA DE MEDIO AMBIENTE Y RECURSOS NATURALES (2001), Ley general del equilibrio ecológico y la protección ambiental, Tomo II, Porrúa, México.

THOMPSON, D. (2002), Tools for environmental management, New Society Publishers, Canada.

TRIST, E. (1992), "A Concept of Organizacional Ecology", en Classics of Organization Theory, compilado por Jay M. Shafritz y Ott J. Steven, 3a. ed., Brooks Cole Publishing Company, Beltmont, California, pp. 316-328.

VALENZUELA CERTUCHA, A. (2003)", Contralor de la planta AMP Amermex, agosto-septiembre, Hermosillo.(A) 